\title{
3D models related to the publication: Size Variation under Domestication: Conservatism in the inner ear shape of wolves, dogs and dingoes
}

\author{
Schweizer Anita V1 , Lebrun Renaud ${ }^{2}$, Wilson Laura A. B. ${ }^{3}$, Costeur Loïc ${ }^{4}$, Schmelzle Thomas ${ }^{5}$, Sánchez-Villagra \\ Marcelo ${ }^{1 *}$ \\ ${ }^{1}$ Paläontologisches Institut und Museum, Karl-Schmid-Strasse 4, 8006 Zürich, Switzerland \\ ${ }^{2}$ Institut des Sciences de l'Evolution de Montpellier, Université de Montpellier, 34095 Montpellier, France \\ ${ }^{3}$ PANGEA Research Centre, School of Biological, Earth and Environmental Sciences, University of New South Wales, Sydney, NSW 2052, \\ Australia \\ ${ }^{4}$ Naturhistorisches Museum Basel, Basel, Switzerland, 4051 \\ ${ }^{5}$ Bad Wimpfen, Germany, 74206 \\ *Corresponding author: m.sanchez@pim.uzh.ch
}

\section{Abstract}

The present 3D Dataset contains the 3D models analyzed in the following publication: Size variation under domestication: Conservatism in the inner ear shape of wolves, dogs and dingoes. Scientific Reports 7, Article number: 13330, https://doi.org/10.1038/s41598-017-13523-9.

Keywords: cochlea, feralisation, petrosal, semicircular canal, zooarchaeology

Submitted:2017-06-01, published online:2017-10-17. https://doi.org/10.18563/m3.3.4.e1

\section{INTRODUCTION}

The present dataset contains 3D virtual endocasts of 39 modern dogs (Canis lupus familiaris) from 20 different breeds, 21 prehistoric specimens, 24 wolves (Canis lupus) as well as eight dingoes (Canis lupus dingo), see table 1 and figure 1. In this study we assessed the intraspecific variation in the morphology of the inner ear using a semilandmark based geometric morphometric approach.

\section{METHODS}

3D surface models of the left inner ear of the specimens were generated by manually colouring the bony labyrinth in Avizo ${ }^{\circledR}$ 8.0, Avizo® 8.1.0. (FEI Visualization Science Group, Germany) and in Geomagic Wrap ${ }^{\circledR} 2013$ 64-Bit-Version (2013.0.1.1206). If the left inner ear was incomplete, the right inner ear was modelled and mirrored. The 3D surfaces are provided in VTK polydata format, which can be opened with VTK based software (for example ISE-MeshTools, Lebrun, 2014).

\section{ACKNOWLEDGEMENTS}

Grant sponsor: Swiss NF. Grant number: 31003A_169395

\section{BIBLIOGRAPHY}

Lebrun, R. (2014). ISE-MeshTools, a 3D interactive fossil reconstruction freeware. 12th Annual Meeting of EAVP,
Torino, Italy; 06/2014.

Schweizer AV, Lebrun R, Wilson LAB, Costeur L, Schmelzle T, Sánchez-Villagra MR. 2017. Size variation under domestication: Conservatism in the inner ear shape of wolves, dogs and dingoes. Scientific Reports 7, Article number: 13330. https://doi.org/10.1038/s41598-017-13523-9. 
A
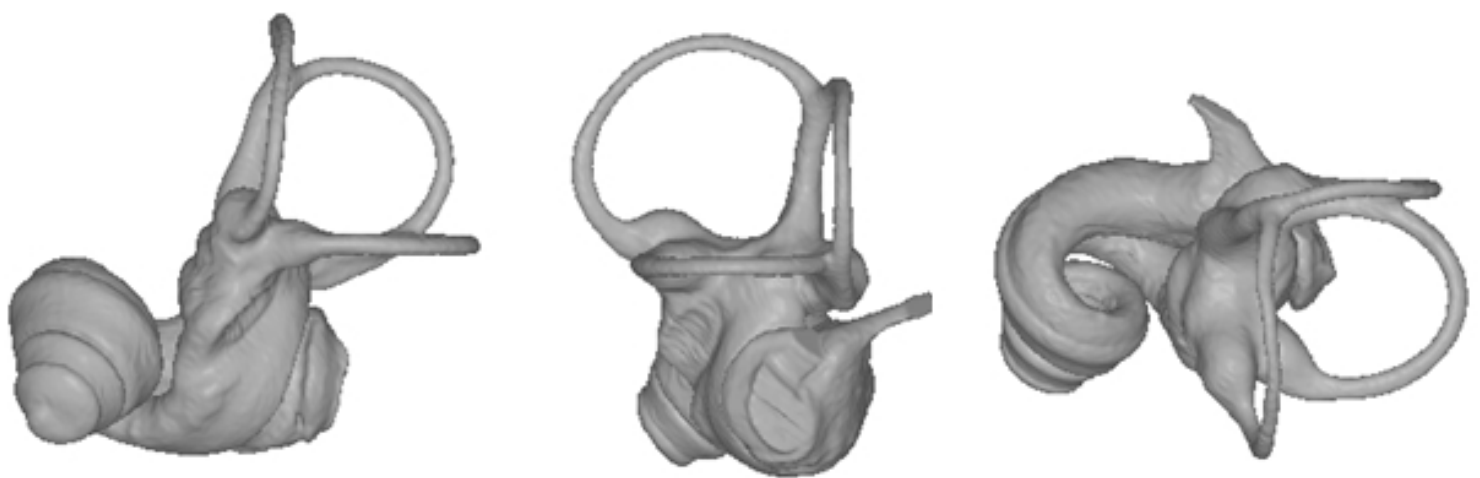

B
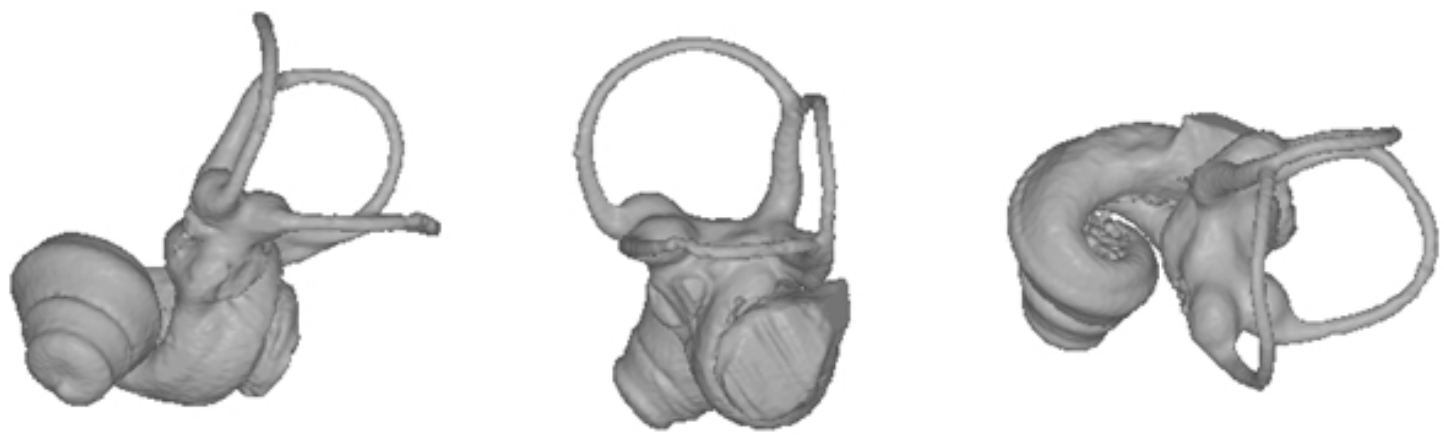

C
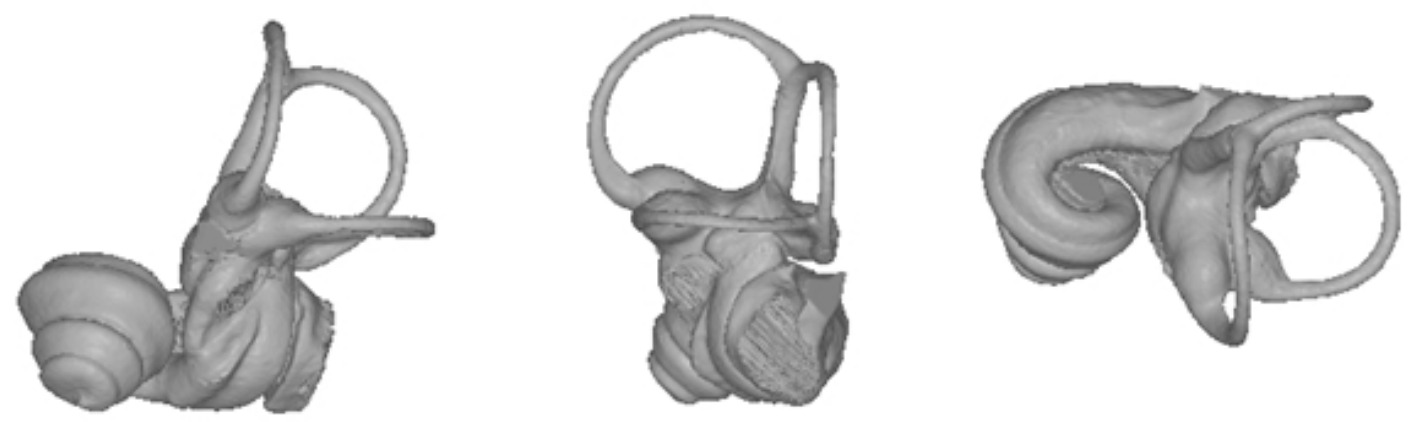

D
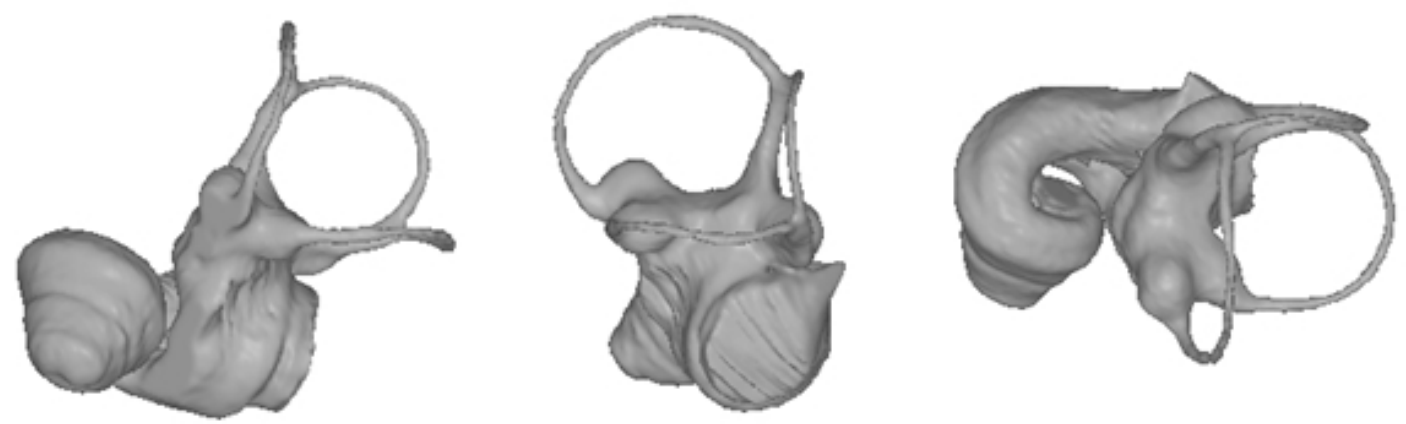

Figure 1. 3D virtual endocast of the left inner ear of A: wolf (ZMUZH 20201); B: prehistoric dog (ZMUZH A.VII.); C: modern dog (PIMUZ A/V 2847, Boxer); D: dingo (M38587) in anterolateral, posterolateral and dorsal view. 


\begin{tabular}{|c|c|}
\hline Model IDs & Taxon \\
\hline M6297 & Canis lupus dingo \\
\hline M24153 & Canis lupus dingo \\
\hline M33608 & Canis lupus dingo \\
\hline M38587 & Canis lupus dingo \\
\hline M39960 & Canis lupus \\
\hline NMB2761 & Canis lupus \\
\hline NMB5258 & Canis lupus \\
\hline NMB8381 & Canis lupus \\
\hline NMB8635 & Canis lupus \\
\hline NMB11019 & Canis lupus arabs \\
\hline NMB12078 & Canis lupus familiaris \\
\hline NMB12079 & Canis lupus familiaris \\
\hline NMB12080 & Canis lupus familiaris \\
\hline NMB12081 & Canis lupus familiaris \\
\hline NMBEA.403. & Canis lupus familiaris \\
\hline NMBEA.5.a. & Canis lupus familiaris \\
\hline NMBC.1362 & Canis lupus lycaon \\
\hline NMBNoNb & Canis lupus occidental \\
\hline NMBSCM320 & Canis lupus \\
\hline NMBZ309 & Canis lupus \\
\hline NMBE1051209 & Canis lupus familiaris \\
\hline NMBE16 & Canis lupus familiaris \\
\hline NMBE1051226 & Canis lupus familiaris \\
\hline NMBE1051381 & Canis lupus familiaris \\
\hline NMBE1051418 & Canis lupus familiaris \\
\hline NMBE-BUR-1057 & Canis lupus familiaris \\
\hline NMBE-DUR-1124 & Canis lupus familiaris \\
\hline NMBE-LAT-1119 & Canis lupus familiaris \\
\hline NMBE-LAT-1136 & Canis lupus familiaris \\
\hline NMBE-LUS-1095 & Canis lupus familiaris \\
\hline NMBE-LUS-1102 & Canis lupus familiaris \\
\hline PIMUZA/V2831 & Canis lupus familiaris \\
\hline PIMUZA/V2832 & Canis lupus familiaris \\
\hline PIMUZA/V2834 & Canis lupus familiaris \\
\hline PIMUZA/V2835 & Canis lupus familiaris \\
\hline PIMUZA/V2836 & Canis lupus familiaris \\
\hline PIMUZA/V2837 & Canis lupus familiaris \\
\hline PIMUZA/V2845 & Canis lupus familiaris \\
\hline PIMUZA/V2846 & Canis lupus familiaris \\
\hline PIMUZA/V2847 & Canis lupus familiaris \\
\hline PIMUZA/V3000 & Canis lupus familiaris \\
\hline PIMUZA/V3001 & Canis lupus familiaris \\
\hline SZ7926 & Canis lupus familiaris \\
\hline SZ7929 & Canis lupus familiaris \\
\hline SZ7930 & Canis lupus familiaris \\
\hline SZ7958 & Canis lupus familiaris \\
\hline SZ7959 & Canis lupus familiaris \\
\hline SZ7961 & Canis lupus familiaris \\
\hline TMMM-150 & Canis lupus familiaris \\
\hline UMZCK.3016 & Canis lupus familiaris \\
\hline UMZCK.3141 & Canis lupus \\
\hline UMZCK.3149 & Canis lupus \\
\hline UMZCK.3150.1 & Canis lupus \\
\hline UMZCK.3152 & Canis lupus \\
\hline
\end{tabular}

\section{Description}

Dingo

Dingo

Dingo

Dingo

Wolf

Wolf

Wolf

Wolf

Wolf

Arabian wolf

Modern dog: Poodle

Modern dog: Poodle

Modern dog: Labrador Retriever

Modern dog: Labrador Retriever

Prehistoric dog

Prehistoric dog

Eastern wolf

Mackenzie Wolf

Wolf

Wolf

Modern dog: Afghan Greyhound

Prehistoric dog

Modern dog: Afghan Greyhound

Modern dog: Akita Inu

Modern dog: Akita Inu

Prehistoric dog

Prehistoric dog

Prehistoric dog

Prehistoric dog

Prehistoric dog

Prehistoric dog

Modern dog: Dachshund

Modern dog: Foxterrier

Modern dog: Appenzeller Mountain Dog

Modern dog: German Shepherd

Modern dog: Boxer

Modern dog: German Shepherd

Modern dog: Dachshund

Modern dog: Boxer

Modern dog: Boxer

Modern dog: Boxer

Modern dog: Foxterrier

Modern dog: Cocker spaniel

Modern dog: Cocker spaniel

Modern dog: Cocker spaniel

Modern dog: Chow chow

Modern dog: Chow chow

Modern dog: Chow chow

Modern dog: Chihuaha

Modern dog (unknown breed)

Wolf

Wolf

Wolf

Wolf
Collection

AM, Sydney

AM, Sydney

AM, Sydney

AM, Sydney

AM, Sydney

NMB, Basel

NMB, Basel

NMB, Basel

NMB, Basel

NMB, Basel

NMB, Basel

NMB, Basel

NMB, Basel

NMB, Basel

NMB, Basel

NMB, Basel

NMB, Basel

NMB, Basel

NMB, Basel

NMB, Basel

NMBE, Bern

NMBE, Bern

NMBE, Bern

NMBE, Bern

NMBE, Bern

NMBE, Bern

NMBE, Bern

NMBE, Bern

NMBE, Bern

NMBE, Bern

NMBE, Bern

PIM, Zürich

PIM, Zürich

PIM, Zürich

PIM, Zürich

PIM, Zürich

PIM, Zürich

PIM, Zürich

PIM, Zürich

PIM, Zürich

PIM, Zürich

PIM, Zürich

ZS, Tübingen

ZS, Tübingen

ZS, Tübingen

ZS, Tübingen

ZS, Tübingen

ZS, Tübingen

TMM, Austin UMZ, Cambridge

UMZ, Cambridge

UMZ, Cambridge

UMZ, Cambridge

UMZ, Cambridge 


\begin{tabular}{|c|c|c|c|}
\hline UMZCK.3221 & Canis lupus dingo Blumenbach & Dingo & UMZ, Cambridge \\
\hline UMZCK.3223 & Canis lupus dingo Blumenbach & Dingo & UMZ, Cambridge \\
\hline UniSydFVS45 & Canis lupus dingo & Dingo & FVS, Sydney \\
\hline UNSWZ354 & Canis lupus dingo & Dingo & UNSW, Sydney \\
\hline ZMUZH10166 & Canis lupus familiaris & Modern dog: Pug & ZM, Zürich \\
\hline ZMUZH10175 & Canis lupus familiaris & Modern dog: Pug & ZM, Zürich \\
\hline ZMUZH10342 & Canis lupus familiaris & Modern dog: St. Bernard & ZM, Zürich \\
\hline ZMUZH10343 & Canis lupus familiaris & Modern dog: St. Bernard & ZM, Zürich \\
\hline ZMUZH13766 & Canis lupus familiaris & Modern dog: Eskimo Dog & ZM, Zürich \\
\hline ZMUZH13854 & Canis lupus Grey & Wolf & ZM, Zürich \\
\hline ZMUZH14842 & Canis lupus familiaris & Modern dog: St. Bernard & ZM, Zürich \\
\hline ZMUZH15858 & Canis lupus & Wolf & ZM, Zürich \\
\hline ZMUZH17118 & Canis lupus & Wolf & ZM, Zürich \\
\hline ZMUZH17210 & Canis lupus occidentalis & Mackenzie Wolf & ZM, Zürich \\
\hline ZMUZH17602 & Canis lupus chanco & Tibetan wolf & ZM, Zürich \\
\hline ZMUZH17603 & Canis lupus chanco & Tibetan wolf & ZM, Zürich \\
\hline ZMUZH17612 & Canis lupus chanco & Tibetan wolf & ZM, Zürich \\
\hline ZMUZH17711 & Canis lupus familiaris & Modern dog: Ibizan Hound & ZM, Zürich \\
\hline ZMUZH17712 & Canis lupus familiaris & Modern dog: Northafrican Greyhound (Sloughi) & ZM, Zürich \\
\hline ZMUZH17713 & Canis lupus familiaris & Modern dog: Russian greyhound (Borzoi) & ZM, Zürich \\
\hline ZMUZH17714 & Canis lupus familiaris & Modern dog: Caucasian Shepherd Dog & ZM, Zürich \\
\hline ZMUZH17715 & Canis lupus familiaris & Modern dog: Caucasian Shepherd Dog & ZM, Zürich \\
\hline ZMUZH17717 & Canis lupus familiaris & Modern dog: Maremma Sheepdog & ZM, Zürich \\
\hline ZMUZH18082 & Canis lupus chanco & Tibetan wolf & ZM, Zürich \\
\hline ZMUZH20201 & Canis lupus chanco & Tibetan wolf & ZM, Zürich \\
\hline ZMUZH20202 & Canis lupus chanco & Tibetan wolf & ZM, Zürich \\
\hline ZMUZHA.6. & Canis lupus familiaris & Prehistoric dog & ZM, Zürich \\
\hline ZMUZHA.II. & Canis lupus familiaris & Prehistoric dog & ZM, Zürich \\
\hline ZMUZHA.IV. & Canis lupus familiaris & Prehistoric dog & ZM, Zürich \\
\hline ZMUZHA.VI. & Canis lupus familiaris & Prehistoric dog & ZM, Zürich \\
\hline ZMUZHA.VII. & Canis lupus familiaris & Prehistoric dog & ZM, Zürich \\
\hline ZMUZHA.VIII. & Canis lupus familiaris & Prehistoric dog & ZM, Zürich \\
\hline ZMUZHEz.2. & Canis lupus familiaris & Prehistoric dog & ZM, Zürich \\
\hline ZMUZHEz.E. & Canis lupus familiaris & Prehistoric dog & ZM, Zürich \\
\hline ZMUZHF.48. & Canis lupus familiaris & Prehistoric dog & ZM, Zürich \\
\hline ZMUZHTerp.1. & Canis lupus familiaris & Prehistoric dog & ZM, Zürich \\
\hline ZMUZHWe.6. & Canis lupus familiaris & Prehistoric dog & ZM, Zürich \\
\hline ZMUZHWyn.9. & Canis lupus familiaris & Prehistoric dog & ZM, Zürich \\
\hline
\end{tabular}

Table 1. List of all the specimens included in the study and the institution they are housed at. AM, Sydney: Australian Museum, Sydney, Australia. FVS, Sydney: University of Sydney, Faculty of Veterinary Science, Sydney, Australia. NBM, Basel: Naturhistorisches Museum Basel, Switzerland. NMBE, Bern: Naturhistorisches Museum der Burgergemeinde Bern, Switzerland. PIM, Zürich: Palaeontological Museum of the University of Zürich, Switzerland. TMM, Austin: Texas Memorial Museum, Austin, Texas, USA. UMZ, Cambridge: University Museum of Zoology, University of Cambridge. UNSW, Sydney: University of New South Wale, Sydney, Australia. ZM, Zürich: Zoological Museum of the University of Zürich, Switzerland. ZS, Tübingen: Zoologische Schausammlung, Universität Tübingen, Germany. 